\section{Want to do clinical research? Changes in the NHS mean there's never been a better time}

\author{
T. Renton, ${ }^{1}$ J. Bagg, ${ }^{2}$ P. G. Robinson ${ }^{3}$ and J. Steele ${ }^{4}$
}

IN BRIEF
- Discusses recent changes in the NHS
research support system.
- Evaluates the transparency of
mechanisms to reimburse NHS
organisations including dental practices
that take part in research and train
clinicians in research skills.
- Considers NHS investment in 'translational
research' to deliver improved and more
cost effective patient care.

This article aims to explain the recent changes in the NHS research support system as it applies to dentistry; information that will be of value to all clinical academics and dentists considering taking part in research. The changes mean that investment goes where patient benefit research is happening. The system has put in place transparent mechanisms to reimburse NHS organisations including dental practices that take part in research and train clinicians in research skills. Through NHS investment in 'translational research', public funds are used directly to deliver improved and more cost effective patient care.

\section{INTRODUCTION}

In the past many dentists have regarded research as esoteric and of no relevance or interest to them. However, dentists from all branches of the profession are now getting involved. They recognise that working to improve patient care is part of being a professional. What's more, the rewards are also direct. Not only does research bring long-term benefits, but the improved systems that form part of the endeavour can also enhance the experience for patients not participating directly. For a lot of dentists, taking part in research will add interest to their work. Put another way, it could even rejuvenate a tired practitioner! The good news is that the opportunities and even the funding to participate in primary care research have never been greater.

\footnotetext{
${ }^{1 *}$ Chair Oral Health London South CLRN and Professor Kings College London, Department of Oral Surgery, Kings College London Dental Institute, Denmark Hill Campus, Bessemer Road, London, SE5 9RS; ${ }^{2}$ Scotland Representative, UKCRN Oral and Dental Specialty Group and Professor of Clinical Microbiology and Head of School, University of Glasgow Dental School, 378 Sauchiehall Street, Glasgow, G2 3JZ; ${ }^{3}$ Deputy Dean, Chair, South Yorkshire Priority Group for Oral and Dental Research and Professor of Dental Public Health, School of Clinical Dentistry, Claremont Crescent, Sheffield, S10 2TA; ${ }^{4}$ Chair UKCRN Oral and Dental Specialty Group and Professor Primary Dental Care, School of Dental Sciences and Centre for Oral Health Research, Newcastle University, Framlington Place, Newcastle upon Tyne, NE2 4BW

${ }^{*}$ Correspondence to: Dr Tara Renton

Email: tara.renton@kcl.ac.uk
}

Accepted 6 January 2012

DOI: 10.1038/sj.bdj.2012.665

${ }^{\circledR}$ British Dental Journal 2012; 213: 105-109
Clinical research, be it dental or medical, is now an expensive, competitive, complicated and highly professional business. The portfolio of clinical research in the UK is vast, incorporating everything from drug trials to qualitative research. In Britain, the NHS plays a key role in delivering almost all of this clinical research and its facilities host virtually every research project that directly involves patients.

In 2006, in recognition of the size and economic importance of this body of work, the Government set up the National Institute for Health Research (NIHR) to oversee and to try to support this vast portfolio of activity in England. However, NIHR is a big and complicated organisation and its structure and mechanisms to support research require some introduction if practitioners are to benefit from them. The devolved nations have parallel but slightly different structures and these will be referenced throughout the article.

This article aims to explain the recent changes in the NHS research support system as it applies to dentistry and will discuss the welcome acceleration in the number of high quality clinical studies being added to the portfolio and how this is overseen by dentists.

\section{ECONOMIC BACKGROUND}

With the switch in emphasis of the UK economy being based less on manufacturing and increasingly on finance and biomedical innovation, the treasury identified the potential of the NHS as the largest clinical trial facility in the world. In 2008 the UK Health Research income was approximately $£ 7.4$ billion (75\% industrial and 75\% health specific). This is around three times the annual spend on dental services in England. It is not just that a lot of money was spent - Britain is actually good at research, particularly medical research, and this is now big business. Consequently NIHR was set up on the premise that it was about both health and wealth. The importance of this area is illustrated by government protection of the NIHR budget despite widespread cuts elsewhere in the public sector.

The UK economy stands to benefit in two important ways:

- By using the NHS as an environment for the development of new services, treatments, products and technologies that become part of our economic output, either by developing from scratch or working with industrial partners

- By adopting techniques and technologies that improve health, so reducing the growing NHS burden and leading to more efficient use of public resources.

In the past a research and development levy was paid annually to NHS organisations to support their research, with minimal justification of activity. In 2006, the NIHR Clinical Research Network was set up to take central control of these funds as a single 
pot and to establish mechanisms to identify and encourage verifiable patient centred research. Specifically, its remit is to use this $£ 1$ billion budget to develop research capacity that will improve health and healthcare.

NIHR has a structure of four 'arms', each performing a complementary function (Fig. 1):

- Research projects, programmes and units direct funding to an 'innovation pathway' for NHS research (Fig. 2)

- The faculty arm develops research expertise through the integrated academic training pathways led by the NIHR Trainees Coordinating Centre to develop the future clinical research workforce

- Research infrastructure provides places and people to support the research via the Clinical Research Network

- Research systems include managing the portfolio of work as well as systems to manage ethics and other permissions.

Each of these arms is described in detail below, although the functions overlap considerably so there is rarely a clean split between the various activities.

\section{Research projects and programmes. NIHR research grants and the 'innovation pathway'}

This arm is a partnership between NIHR and the leading research councils to fund research in the form of grants. It identifies funding for patient-centred research with emphasis on improving patient care and health. Partnership is necessary because the 'innovation pathway' between basic and laboratory research and widespread implementation involves five stages: creating the idea, refining it, evaluating it, appraising it and then using it to improve health; with each stage suited to different funding agencies (Fig. 2).

The research councils (particularly the Medical Research Council) support the early stages, predominantly laboratorybased research. NIHR schemes (in blue in Figure 2) then fund research projects, programmes and schemes that are closer to patients, with a view to translating the best theoretical or biomedical research into benefits for patients. These funding programmes include the health technology assessment (HTA) for trials and evidence syntheses that

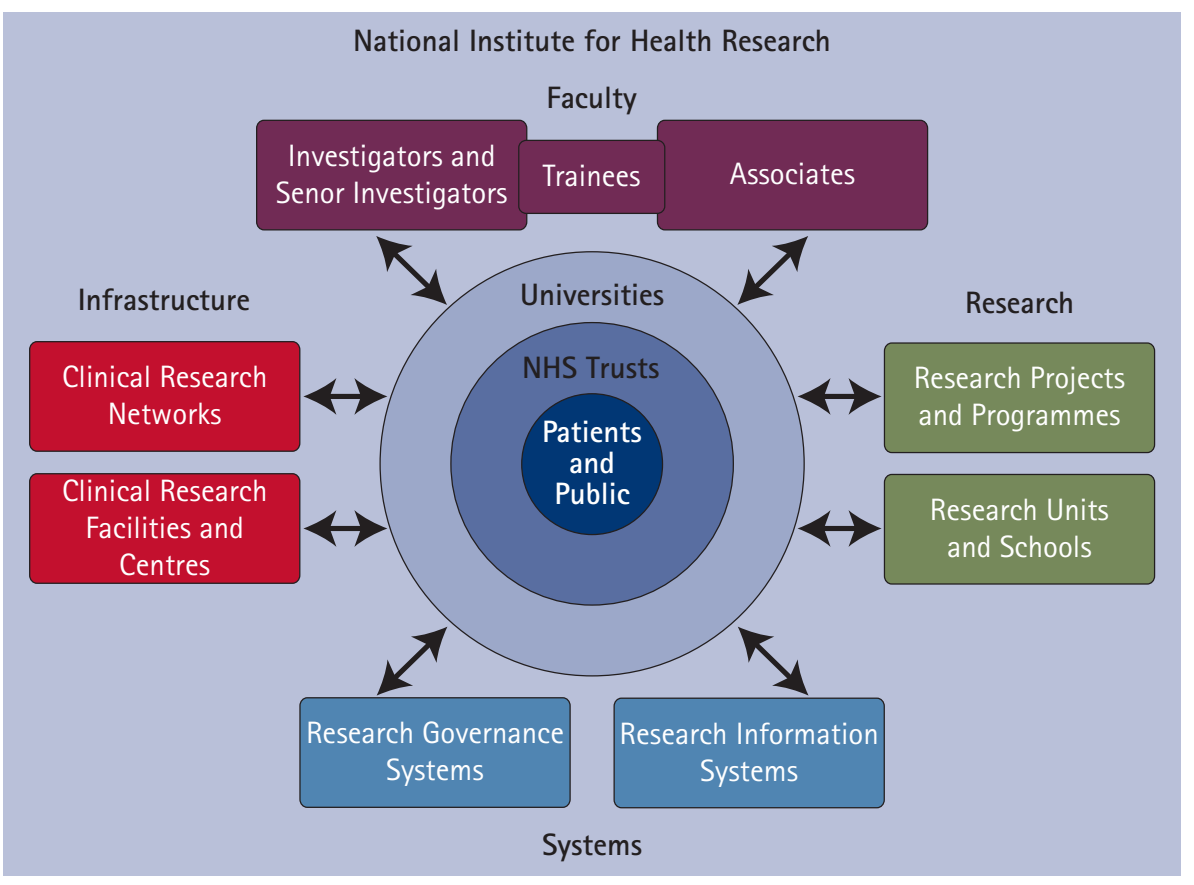

Fig. 1 Structure of NIHR

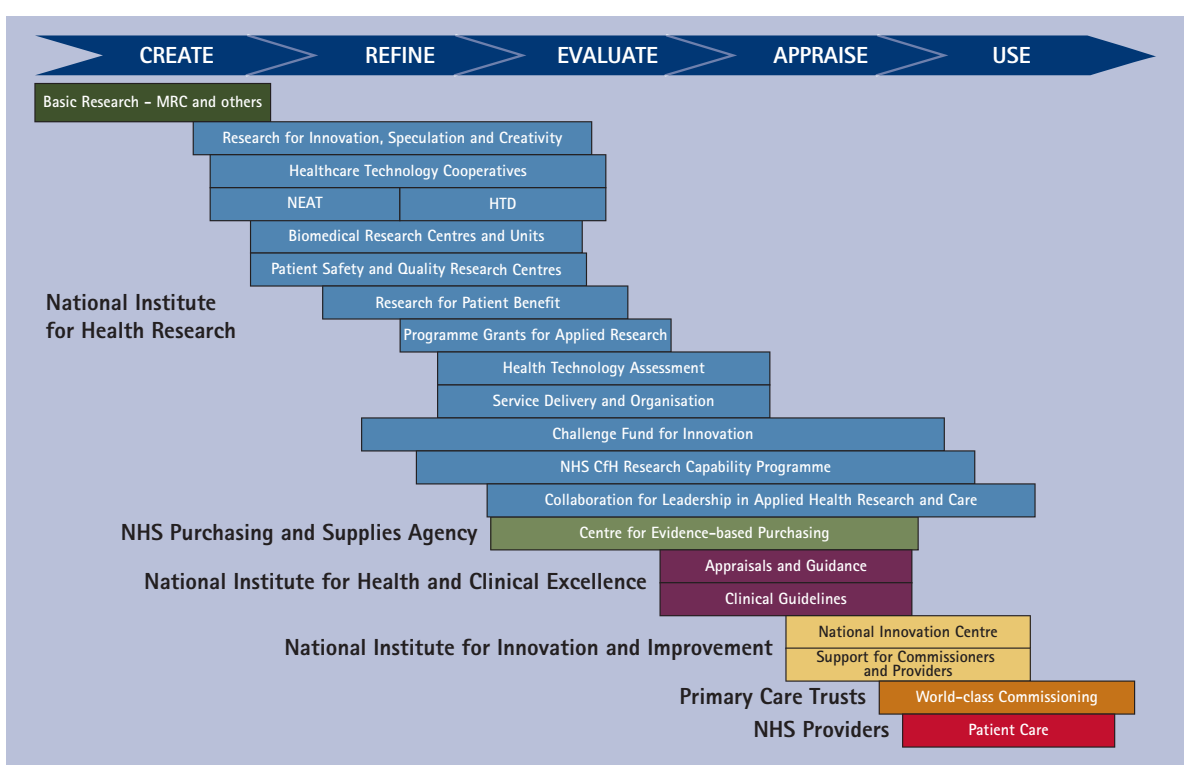

Fig. 2 The central role of NIHR research in the innovation pathway

evaluate 'technologies' in their broadest sense. Several very large dental trials are now funded by HTA. These studies are based in dozens of dental practices and answering important questions about dental treatment to determine what works and what does not. A good example is the FiCTION trial (http:// www.hta.ac.uk/1783), which will ascertain whether fillings are an effective treatment for caries in the deciduous dentition.

Beyond NIHR on the innovation pathway are funding streams for the final stages of putting robust research into practice. We are all familiar, for example, with NICE (National Institute for Health and Clinical Excellence) guidelines and their application. At the end of the pathway are the NHS health care providers: dentists, doctors, nurses, opticians and many others, who use evidence and training to provide excellent care.

\section{Research faculty: research training and NIHRTCC}

Research is not an ad hoc activity for amateur enthusiasts but a highly specialised activity for which training and experience are critical. Great clinicians are not always great researchers, and vice versa. But clinical research needs people who are trained and experts in both arenas and who can work with hardcore non-clinical researchers and front-line clinicians. They have to 
deliver both complex research and high quality clinical care.

The NIHR funds for research training aim to build a leading NHS research faculty into the future, to develop research careers, research leaders and collaborators. This research capacity development is managed by the NIHR Trainees Coordinating Centre (NIHRTCC) and funded by the Department of Health. The NIHRTCC gives training awards to researchers whose work focuses on people and patient-based health research. The research must be relevant to the NHS, focused on the current and future needs of patients and the public and expected to have an impact within five years of its completion. The NIHR does not fund basic research or work involving animals and/or animal tissue.

Dentists are eligible for NIHR funded research training. Indeed several dentists across the UK have already benefited. Four schemes are especially relevant to us:

- Academic clinical fellow (ACF) posts are for junior dentists who want to pursue a career in academia. They are normally linked with a speciality training programme in a dental hospital, but potentially also in primary care. The scheme will pay for 25\% of a salary, usually of a specialist registrar, for three years to allow for time away from service and training so that the fellow can work up an application for $\mathrm{PhD}$ funding. There are several specialty trainees around the country in ACF posts. Unfortunately, primary dental care lacks formal training programmes to which ACFs can be linked after vocational training. However, there are schemes for general medical practitioners and there are now attempts to formalise the same route for GDPs

- Pre-doctoral fellowships represent the natural progression from an ACF, allowing the fellow to take three years to complete a $\mathrm{PhD}$. The fellowships are competitive but a young general dental practitioner who has undertaken an ACF would be well placed to apply for one if he or she had strong academic supervisors and a good project. Compared with most PhD posts, pre-doctoral fellowships are well-funded

- Post-doctoral fellowships are the next step for someone who has completed a $\mathrm{PhD}$ and who has extensive clinical experience in a speciality, or potentially in general medical or dental practice. The award most relevant to dentists is the clinician scientist award. These are fiercely competitive but provide five years funding for a programme of research. Applicants should have publications and ideally, other research grants. Most are close to consultant level. Although there are only a few clinician scientists at present, they do include dentists

- In-practice fellowships are aimed at experienced doctors and dentists in primary care who have done some research and want to take it further. They are well funded and offer a chance for primary care dentists at any age to get into research. Good academic support is required, but again dentists have been successful.

\section{Research infrastructure: \\ facilities, support staff and the research networks}

Clinical research often incurs costs that research grants do not cover. There need to be buildings, facilities and people in the background so that research can happen. It is important that organisations and practices that conduct research receive support for this, rather than being expected to carry the cost themselves.

For example, a dental practice taking part in research still has to pay for electricity, nurses and perhaps additional work, such as extra radiographs. Practices will find it hard to maintain their enthusiasm if these costs are not reimbursed. Outside of dentistry, some research requires highly specialised facilities with state of the art imaging systems or other equipment that is used for many different trials.

The Clinical Research Network (CRN) was set up to allocate and manage NIHR funds to meet these NHS service support costs. The networks are discussed in detail in the next section, but first it is important to think about which projects are eligible for funding, so that infrastructure money is targeted on the right projects and places. In England, the Department of Health has established the eligibility criteria for CRN support. Applications for CRN support of non-commercial studies are made through the NIHR coordinated system for gaining
NHS permission (NIHR CSP) via the integrated research application system (IRAS). Applications for commercial studies can be made either through this same route or via direct contact with one of the eight NIHR clinical research networks.

Eligible studies are included on the NIHR clinical research network portfolio and recorded on a public, searchable database: the portfolio database. This database captures activity data and provides analysis tools to enable active management of current studies run within the CRN. Activity data from portfolio studies are used to inform the allocation of NHS infrastructure for research (including NHS support costs) to the comprehensive local research networks and supports the performance management of the CRN. In this way support can be directed to where patients are recruited and what is needed locally for successful study delivery. The devolved nations have equivalent arrangements for this distribution of resource.

Some studies automatically go on to the portfolio because of the very robust nature of decision making when they are awarded by the funding body. Research councils look very carefully at protocols before handing over money. Other funding agencies are more variable, so there are guidelines about what is and is not eligible. A link to the criteria for adoption on to the NIHR CRN portfolio is provided at the end of this article.

Another key part of the infrastructure development has been the Research Design Service (RDS), which supports researchers to develop and design high quality research proposals for submission to NIHR and other national, peer-reviewed funding competitions. The RDS gives researchers expert advice on all aspects of preparing grant applications for applied research in health and social care, including statistics, research techniques, clinical trials, evidence synthesis, health economics, epidemiology, public and patient involvement, ethics and governance. There are ten NIHR research design services, one in each strategic health authority area.

\section{Information systems and governance}

Finally, with so much research activity, training and infrastructure, NIHR requires information systems to focus resources at 
the right places and to 'join-up' the whole system to do its job.

Two parts to these systems are relevant to dentists taking part in research:

- The networks described already and the portfolio which they oversee and use

- The systems to ensure that ethics and governance procedures are handled efficiently.

\section{More about networks}

The UK departments of health fund clinical research networks in all four nations. Together these national networks form the UK Clinical Research Network (UKCRN). The structure of the networks varies by country, but their aim is common: to provide the infrastructure to support high quality clinical research for the benefit of patients. The clinical research networks across the UK work together in an integrated fashion to promote partnership and UK-wide working wherever possible.

The NIHR Clinical Research Network Coordinating Centre (NIHR CRN CC) has bases in Leeds and London. This organisation uses the NIHR portfolio, not just to ensure money goes to the right places, but as a means to performance manage all clinical research in England and the networks that look after it. The other three devolved nations operate in a slightly different way, but all have arrangements to ensure that money follows research patients.

The network is set up both geographically and by subject area. The geographical divisions ensure that money is correctly assigned locally, in proportion to activity, as described later. The other dimension of the network is divided by clinical specialty.

\section{Topic networks and specialty groups}

Initially there were six topic specific clinical research networks for the big national priorities for clinical research such as cancer, stroke and so on. These original clinical networks were hugely successful in enabling trials to run nationally with large increases in patient recruitment, which they achieved by providing dedicated research staff and other resources to work with NHS clinical teams. Soon after this a Primary Care Research Network was established to fulfil the same role in primary care studies. This network is comprised of eight local research networks that cover the whole of England and interact closely with the topic specific networks. Primary care dentistry comes under its portfolio and there has been close liaison with the Oral and Dental Specialty Group (see below).

This network concept was seen as a good model for developing research and thus in 2007 the Comprehensive Clinical Research Network was established to encompass all of the other specialities (another 24 groups), including 'oral and dental'. The role of these groups is to oversee the portfolio by advising on which studies are adopted, tracking them and detecting recruitment problems early, then using the network to identify extra sites or support so that the studies complete on time.

\section{THE ORAL AND DENTAL SPECIALTY GROUP}

The oral and dental group has overseen a rapid transformation in portfolio research in its area. From a starting point in early 2009 with almost no portfolio studies, there are now 38. Some examples of adopted studies are provided in Table 1. This number is still small by comparison with other specialities, but the total number of patients recruited into oral and dental portfolio studies is increasing rapidly and our studies tend to recruit on time and target better than most groups. Recently, some large primary care trials involving many hundreds of patients have commenced.

The oral and dental group has also tried to smooth the path for upcoming trials and to organise the workforce to make the UK an attractive place to run oral and dental research. While research in hospitals can be handled in the same way as in any other speciality, GDPs will not need reminding that primary care dentistry has a unique funding and business model, which research funding must recognise. Specifically, the group has developed standardised approaches to studies in primary care so that dental practices are appropriately remunerated through service support costs and patient charges are managed consistently. These discussions have involved the Department of Health, British Society for Oral and Dental Research, primary care groups and researchers and dentists with experience of taking part in big NHS studies. The group has also produced a 'CV' for
Table 1 Examples of studies adopted on to the NIHR portfolio

Monitoring oral cancer patients using novel lab-on-a-chip ensembles

Are micro-implants as effective at providing orthodontic anchorage as conventional methods?

Chewing gum and orthodontic pain

Treatment of periodontitis and metabolic control in patients with type 2 diabetes mellitus

A comparison of pre-medication with oral paracetamol versus intravenous paracetamol at induction for analgesia following wisdom tooth extraction

Evaluating saliva biomarkers to predict clinical periodontal disease

Improvdent: improving dentures for patient benefit - a cross over randomised clinical trial compa ing impression materials for complete dentures

Eliminating periodontal infection in patients with type 2 diabetes

$X$-ray microtomography study of carious teeth and comparison of its removal by various techniques

Interval dental recalls trial (investigation of NICE technologies for enabling risk-variable-adjustedlength dental recall trial)

Seal or varnish? A randomised trial to determine the relative cost and effectiveness of pit and fissure sealants and fluoride varnish in preventing dental decay

Filling children's teeth: indicated or not?

Preventing obesity in young people attending primary dental care settings: an exploratory randomised controlled trial

clinical dental research and has met with industry partners to encourage them to fund dental research in the UK.

\section{GEOGRAPHICAL NETWORKS}

\section{England}

As mentioned earlier, the NIHR clinical research network is made up of eight networks that provide infrastructure support for all disease areas across England.

The Oral and Dental Speciality Group is part of the Comprehensive Clinical Research Network, which is divided into 25 local networks that are equivalent in size to clusters of 6-10 PCTs and map to existing NHS borders. Local networks receive central money in proportion to the clinical research activity in their area (measured by the number of patients recruited), and distribute it accordingly. Nine CLRNs have identified oral and dental research as a priority area (Table 2).

\section{Northern Ireland}

The Health and Social Care (HSC) R\&D Office has established the Northern Ireland Clinical Research Network (NICRN) to 
Table 2 Membership of the NIHR National Group for Oral and Dental Research and the CLRN or devolved nation represented*

\begin{tabular}{l|l|l}
$\begin{array}{l}\text { Birmingham and } \\
\text { the black country }\end{array}$ & Prof Thomas Dietrich & t.dietrich@bham.ac.uk \\
\hline $\begin{array}{l}\text { Central and } \\
\text { East London }\end{array}$ & Prof John Galloway & joh.galloway@uclh.nhs.uk \\
\hline Cheshire and Merseyside & Prof Susan Higham @liverpool.ac.uk \\
\hline Greater Manchester & Prof Martin Tickle & Martin.tickle@northwest.nhs.uk \\
\hline Kent and Medway & Prof Ken Eaton & keaton@rcseng.ac.uk \\
\hline London (North West) & Mr Manolis Heliotis & Manolis.heliotis@nwlh.nhs.uk \\
\hline London (South) & Prof Tara Renton & Tara.renton@kcl.ac.uk \\
\hline $\begin{array}{l}\text { Northumberland, } \\
\text { Tyne and Wear }\end{array}$ & Prof Jimmy Steele (Chair) & jimmy.steele@newcastle.ac.uk \\
\hline Peninsula & Prof David Moles & David.moles@pds.ac.uk \\
\hline South Yorkshire & Prof Peter Robinson (Deputy Chair) & Peter.g.robinson@sheffield.ac.uk \\
\hline Western & Dr Anthony Ireland & Tony.ireland@ruh-bath.nhs.uk \\
\hline West Yorkshire & Dr James McCaul & $\begin{array}{l}\text { James.mccaul@bradfordhospitals.nhs.uk } \\
\text { Prof Jeremy Bagg and } \\
\text { (currently Tim Watson), Primary Care Research Networks (currently Terri Harding) and Departmentof Health (currently Sue Gregory) }\end{array}$ \\
\hline Scotland & Prof Stephen Richmond & $\begin{array}{l}\text { Jeremy.bagg@glasgow.ac.uk } \\
\text { j.e.clarkson@dundee.ac.uk }\end{array}$ \\
\hline Wales & richmonds@cardiff.ac.uk \\
\hline
\end{tabular}

provide research infrastructure as part of the UKCRN. This managed, comprehensive network, based at the Belfast Royal Hospitals site, encompasses nine NICRN areas of interest. These are networks of clinicians and other professionals with research interests in specific diseases and access to sufficient patients to enable recruitment. The NICRN maintains a portfolio of studies and assists with setting them up.

\section{Scotland}

The Scottish Government Chief Scientist Office (CSO) has established five topic-specific clinical research networks in Scotland to complement the primary care and cancer networks that already existed. These networks, which form part of the UK Clinical Research Network, provide the infrastructure to support high quality studies across many areas of disease and clinical need. The CSO has encouraged NHS Scotland and the clinical academic community to expand its clinical research collaboratively with partner networks across the UK.

\section{Wales}

The Wales Office of Research and Development for Health and Social Care (WORD) has established the Clinical Research Collaboration Cymru (CRC Cymru). The aim of CRC Cymru is to provide dedicated infrastructure to improve the quality, quantity, coordination, integration, inclusiveness and speed of health and social care research in Wales. CRC Cymru works closely with other research networks and key partners across the UK. For example, the Welsh Cancer Trials Network is an umbrella organisation for clinical cancer research in Wales. In addition, there are nine multi-disciplinary research networks. CRC Cymru also hosts research infrastructure and technical support groups, specialist professional trial units and a coordinating centre in Cardiff with professional research support staff. They facilitate engagement with industry; manage the CRC Cymru portfolio, CRC Cymru service user and carer network and deliver a national training programme.

\section{European Clinical Research Infrastructures Network}

In the same manner in which the UK is developing its infrastructure to support high quality clinical trials, many European countries are engaged in a similar exercise. A European Clinical Research Infrastructures Network (ECRIN) has been established to facilitate closer working of EU member states in this area. This EU-funded programme is a sustainable, not-for-profit infrastructure supporting multinational clinical research projects in Europe. It aims to integrate national clinical research facilities into a panEuropean infrastructure, able to provide support to multinational clinical research in any medical field and for any category of clinical research.

\section{CONCLUSION}

This article has outlined how the NIHR is supporting clinical research in the NHS by funding and training staff via clinical research networks and by building the right infrastructure. The NHS could be the biggest and the best 'laboratory' to answer the largest and most pressing problems in dentistry. A number of dentists are already taking advantage of NIHR support and training and many more could do the same.

Existing clinical academics will recognise that much of the funding for this is competitive and so will need to collaborate to devise the strongest proposals. Dentists not already participating in research will have experience of real-life problems and should contact others with the relevant expertise at their nearest dental school or at any of the other centres listed to find out how they might get involved. Contact details of the local priority groups for oral and dental research are given in Table 1 .

\section{CONTACTS}

\section{NIHR resources}

- Comprehensive Clinical Research Network team: crncc.comprehensive@ nihr.ac.uk

- Criteria for eligibility for adoption onto the portfolio: www.crncc. nihr.ac.uk/about_us/processes/ portfolio/p_eligibility

- Research design service: www.nihr.ac.uk/ infrastructure/Pages/research_design_ service.aspx

- Trainees coordinating centre (NIHRTCC): www.nihrtcc.nhs.uk

\section{Dental schools and other organisations already involved in oral and dental research}

- www.dentalschoolscouncil.ac.uk/ uk_dental_schools_list.htm

- Faculty of General Dental Practice (UK): www.fgdp.org.uk/research.ashx

- European Clinical Research Infrastructures Network (ECRIN): www.ecrin.org 